\title{
REQUEST BASED \\ VIRTUAL ORGANISATIONS (RBVO): AN IMPLEMENTATION SCENARIO
}

\author{
Bob Roberts \\ Centre for Applied Research in Information Systems \\ Kingston University, $U K$ \\ R.Roberts@kingston.ac.uk
}

Adomas Svirskas, Brian Matthews

Business and Information Technology Department CCLRC Rutherford Appleton Laboratory, UK

\{A.Svirskas, B.M.Matthews\}@rl.ac.uk

\begin{abstract}
Evolving e-commerce technologies increasingly enable organisations to participate in different types of network forms or in electronic markets with previously unidentified trading partners. Virtual organisations (VO) take different forms, have varying lifecycles and involve different scope and depth of relationships. This paper examines the literature in terms of the terminology of virtual organisations, the business drivers, the common theoretical concepts and models as well as the enabling technologies. A specific form of $V O$, Request Based Virtual Organisation ( $R B V O$ ), is then considered in relation to these VO variants, particularly as realised through the practical work done within the framework of the EU sponsored LAURA project that facilitates interregional zones of adaptive electronic commerce.
\end{abstract}

\section{INTRODUCTION}

The literature presents a common theme of increasing competitive pressures on companies exacerbating the need for greater flexibility, efficiency, responsiveness and innovation. The common theme is one of traditional external boundaries of organisations beginning to blur, often with e-commerce as a key enabler of such change. Such links may take different forms, have varying lifecycles and involve different scope and depth of relationships. The separation between internal and external processes becomes less clear as inter-organisational systems facilitate more co-ordinated exchange and sharing of information and this may involve the innovative use of e-commerce technologies for new organisational arrangements, value acceleration and new value added processes.

Request based Virtual organisations (RBVOs) are a special kind of Virtual Organizations, and comprise a cluster of partnering organizations that have totally replaced their vertical integration into a virtual one. By their nature, RBVOs are short-lived entities that are formed with the identification of a business opportunity offered by electronic commerce. This introduction is followed by Section 2 which 
examines the business drivers, the common theoretical concepts and models as well as the enabling technologies for VOs. In section 3, the RBVO concept as realised through the EU sponsored LAURA project is introduced and compared to the VO characteristics identified in section 2 . Section 4 then expands on the technical architecture chosen to support the RBVO concept in the LAURA context while section 5 concludes with the outcomes of the LAURA project, the realisation of the benefits and the future research ideas. These ideas include trust and security aspect in VO management related areas and are based on choreographed B2B interaction models.

\section{A REVIEW OF VIRTUAL ORGANISATIONS (VOs)}

\subsection{The Business Drivers}

A recurring theme in the earlier inter -organisational system (IOS) literature is the role of IT as a key enabler for competitive advantage through cementing relationships with customers and by enabling integration forwards or backwards in the industry value chain (Cash and Konsynski 1985, Cash 1985, Johnston \& Vitale 1988). Benjamin and Malone (1986) also noted that through electronic networks firms may achieve an integration effect by tightly coupling processes at the interface between stages of the value chain.

More recent literature suggests that the broad concept of the virtual organisation has accelerated with the emergence of Internet / Web based communities of common interest and that enterprises are aligning within a series of 'value networks' against other groups of enterprises (Bovet \& Martha 2000, Bressler \& Grantham 2000 ). The evolution of the B2B Internet environment will increasingly provide the means to integrate supply chain practices and inter-organisational trading processes. Shorter product life cycles, more intensive competition, faster technological change and more specialised markets have led to various kinds of inter-organisation agreements, collaborations and partnerships as firms join together to create partnerships and also incorporate small firms in recognition of their capability for flexibility, responsiveness and innovation (Davidow \& Malone 1992, Rhodes \& Carter 1994, Hagel \& Armstrong 1997, Aldrich 1999).

\subsection{Virtual Organisation Terminology}

A virtual organisation (VO) is described in many cases as a network among organisations while others argue that VOs should not be viewed solely as networks among organisations but as a radical approach to management, or a strategic approach that leads to dynamically re-configurable enterprises (Sieber \& Griese 1999, Saabeel et al 2002). Lethbridge (2001) observes that while a member may exercise more power than others within the VO, each member operates as both a member of a VO and as an independent organisation. Lethbridge also notes that each member of a VO may also be a member of one or more other VOs.

There is, however, a lack of consistent terminology in the various discussions of organisational networks in the literature, with terms such as virtual networks, strategic networks, dynamic networks, extended networks and value networks. 
These different terms are often used as synonyms but sometimes also indicate different characteristics related to longevity, purpose and culture. Nikoleris and Johansson (2003), for example, differentiate between virtual and extended enterprises as two emerging forms of dynamic networked organisations. Virtual enterprises are described as temporary consortiums of independent member companies who come together to exploit a particular market opportunity while the focus of extended enterprises is on longer term collaborative alliances. However, the success of both relies heavily on the seamless and effectively facilitated information flow between the participating enterprises and the ability to analyse, measure and improve communication.

Timmers (1999) also differentiates between value networks and dynamic markets where the former is characterised by a limited number of long term relationships while the latter involves many relationships over shorter time scales in order to maximise product, price or delivery configurations by selecting appropriate business partners. Marjanovic (2002, p.713), on the other hand, coins the term 'dynamic virtual enterprises' that involves rapid teaming of business partners (in particular small and medium sized enterprises) in pursuit of specific business objectives. "Business partners are linked dynamically (on demand) according to the requirements made by the customer. Thus, partners collaborate on a short term basis (during the VE lifecycle) to solve a particular business problem. Once the problem is solved cooperation ends and the virtual enterprise ceases to exist."

\subsection{Technologies for Enabling Virtual Organisations}

Silva et al (2003), in reference to the implementation of what they refer to as the agile/virtual enterprise (A/VE) model, argue that two main inter related aspects must be met; dynamic reconfigurability and business alignment of the $\mathrm{A} / \mathrm{VE}$ with the market requirements. In reviewing the offerings of key e-marketplace makers they noted that while all of them still supported EDI, Web Services and ebXML are the most promising technologies for the creation of dynamic collaborative environments and business process integration.

Choudhury (1997) examined the evolving issue of inter-organizational information systems from the standpoint of a firm making strategic decisions about inter-organisational (IOS) development. Choudhury addressed the questions of what types of IOS might be useful, and how those IOS might be developed. Three types of IOS are described: electronic monopolies (e.g. supporting a sole source relationship for a product), multilateral IOS (e.g. communicating with a large number of trading partners over a single logical inter-organisational link and electronic dyads (e.g. EDI links between buyers / sellers). However, the increasing feasibility of adopting a peer to peer (P2P) approach for B2B e-commerce offers a new IOS option. Lee (2004), for example, argues that the need for centralised exchanges decreases through P2P networks and that a P2P architecture offers advantages over exchange based models including avoidance of fees charged by exchanges, reduction in the complexity and expense of networking and the scalability of P2P networks. Lee goes on to suggest that P2P e-commerce can be viewed as a 'dynamic electronic dyad' from the IOS perspective where the buyer/seller dynamically establishes individual logical links with each of a dynamically selected number of sellers/buyers. 


\section{RBVO IN RELATION TO THE LITERATURE}

The RBVO concept encapsulates many of the characteristics described in the broad range of VO literature i.e. the flexible and dynamic reconfigurability of independent companies who come together to leverage on the complementarity of their competencies, products and services to meet market needs. However, while ecommerce may mean that asking for quotes from an increasing number of sellers can mitigate ignorance of the price of a very well defined product, overcoming ignorance of product quality and other supplier capabilities may be more difficult. The RBVO is coupled with the concept of sector specific Service Level Agreements (SLA) to address this issue. Furthermore, organisations participating in RBVO formations can reduce the costs of market search, and benefit from more effective monitoring schemes thus lowering transaction costs. Improved information flows can also facilitate improved planning and more co-ordinated actions to reduce uncertainty.

The authors of this paper were engaged in a project co-funded by the European Commission entitled "LAURA - Adaptive Zones for Interregional Electronic Commerce based on the concepts of Request-Based Virtual Organizations and sector-specific Service Level Agreements" (LAURA Project 2004). The LAURA project aims to increase the competitiveness and business efficiency of Small and Medium Enterprises (SMEs) from the Less Favoured Regions (LFRs) of Europe, by introducing state-of-the-art electronic commerce in those companies. This project innovates in introducing the concept of Request-Based Virtual Organizations (RBVOs) that are formed using the concept of sector-specific Service Level Agreements between trading partners.

The architecture and technology approach of the LAURA project supports the RBVO concept, builds on opportunities offered by the peer to peer (P2P) approach and utilises the latest e-business standards, such as ebXML. The discovery phase, for example, is implemented using a $\mathrm{P} 2 \mathrm{P}$ approach, as a natural form of discovery behaviour. Subsequent phases of search for particular products and potential partners within the identified domains (matchmaking) as well as business conversations use a more conventional approach that innovate by building on aspects of ebXML such as BPSS and ebXML messaging.

The distinctive characteristics of RBVO as encapsulated in the LAURA project can be summarised as:

- A cluster of geographically dispersed organisations either within regions or inter regionally (typically SMEs).

- Organisations are independent and may belong to different RBVOs simultaneously and at different times.

- A possibility for an enterprise to discover potential business partners upon demand and advertise itself in a standard way.

- A range of relationships from transactional to collaborative that vary dynamically over time in response to market opportunities.

- The P2P architecture provides a flexible topology for virtual formations (JXTA 2004).

- The ebXML standard provides the foundation for messaging and business process management (EBXML 2003)

- Business documents are modelled according to the Open Applications 
Group Integration Specification - OAGIS (Rowell 2002; Flebowitz 2002, Dubray 2001).

- Support Centres provide a low cost and trust oriented environment conducive to SME involvement and thus improve their competitiveness and business efficiency.

- Sector specific SLAs provide a means to analyse and measure the performance of participants.

- Lower transaction costs for geographically dispersed transactions.

\section{THE ARCHITECTURE TO SUPPORT RBVO CONCEPTS IN THE LAURA CONTEXT}

The concept of Virtual Organisation (VO) must be supported by appropriate architectural and technical implementation solutions, as well as suitable operational services, in order to provide its expected value for business partners. In addressing this particular task certain general B2B collaboration aspects were identified which led to the development of a generic architectural framework. Three areas of particular importance were singled out for end-to-end business collaboration; discovery and matchmaking of the business partners, secure and reliable business data transmission and business process specification and enactment.

The discovery and matchmaking aspect of the overall $\mathrm{B} 2 \mathrm{~B}$ problem becomes especially important in the SME e-business context, mainly due to the potential for a great number of collaborative participants, the diversity of their capabilities, the lack of standardisation for product and service description, as well as the absence of mechanisms to harmonise the latter. In order to fully reveal the potential of RBVOs as highly dynamic virtual business formations an innovative approach is taken, which is based on natural trading behaviour pattern, expressing direct interaction between partners. Recent developments in the peer-to-peer (P2P) computing field allow this pattern to be implemented. This approach results in a more flexible topology for virtual formations and bridges the gap between the isolated 'islands' thus forming a business to business grid that widens the possibilities for collaboration and increases their availability to business partners.

In the LAURA network context there are two types of peers:

- Domain Hubs, acting on behalf of SMEs, which are not capable of using LAURA business collaboration service themselves

- Advanced SMEs, using the LAURA collaboration service connected to their back-office and/or ERP systems

Project JXTA provides a simple and generic framework for P2P networking and provides a base P2P infrastructure over which other P2P applications can be built. The JXTA Protocols document describes six XML-based protocols that standardise the methods used by peers to discover each other and interact to form peer groups (JXTA 2004). 


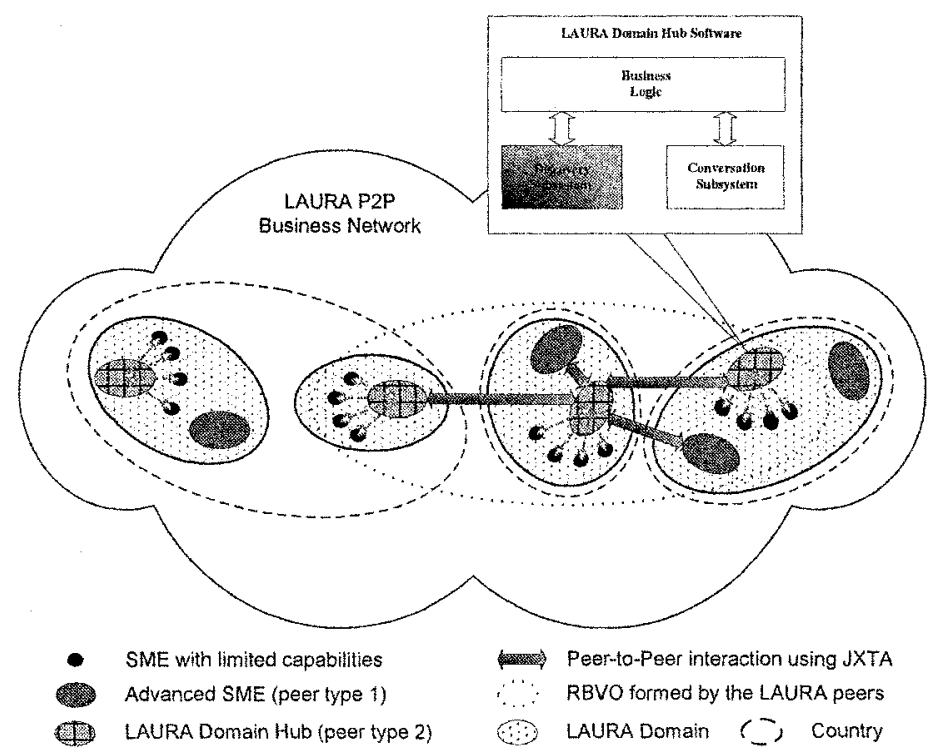

Figure 1. LAURA Virtual Network based of the concept of peer-to-peer interaction builds on JXTA virtual network (JXTA 2004) conceptual structure

The JXTA technology is particularly applicable from the RBVO point of view and especially with regard to the multi-domain nature of the solution. The JXTA Virtual Network, which allows flexible mapping between the physical resources and the logical entities, required for a multi-domain e-business network such as LAURA is shown in Figure 1.

\section{CONCLUSIONS AND FUTURE WORK}

As well as implementing a fully functional software prototype in the five European regions, the Laura Project has successfully carried out the establishment and operation of Support Centres enabling regional SMEs to conduct on-line B2B transactions on an intra and interregional level. This reflects the adoption of innovative notions such as the RBVO concept enabled by technology approaches such as EBXML and P2P to meet SME business requirements. The main objective of the deployment partners was the introduction of the LAURA proposed ecommerce solution in the participating regions, at both the operational and technology level. More specifically, they co-ordinated the introduction of ecommerce zones into selected pilot SMEs and, based on their experience of regional business environment, they evaluated the operation of LAURA proposed ecommerce zones.

The consortium has also provided a concrete dissemination and exploitation strategy for the project results and the commercialization of concepts, methodologies and prototypes developed in the project. The German LAURA partners have set up a spin-off "e-commerce-express.de GmbH I.G" for the 
exploitation of the LAURA solution in Germany, which will offer LAURA services and additional back-end support to already running support initiatives relating to ecommerce (www.ecommerceexpress.de). This LAURA based system is marketed as an adaptable solution and an online collaborative commerce system for SMEs with production to order characteristics. The system automates administrative processes with stakeholders in the supply chain and adds eCommerce functions to extend the boundaries and benefit of an SME's ERP system.

Some of the ideas and experience gained in the LAURA project will be applied in another EU sponsored project related to dynamic VOs - TrustCoM (Dimitrakos 2004), as two of the authors are working now in the TrustCoM project. The main objective of TrustCoM is to provide a trust and contract management framework enabling the definition and secure enactment of collaborative business processes within Virtual Organisations that are formed on-demand, are self-managed and evolve dynamically, sharing computation, data, information and knowledge across enterprise boundaries, in order to tackle collaborative projects that their participants could not undertake individually, or collectively offer services to customers that could not be provided by the individual enterprises.

A novel trust and contract management reference architecture that will enable collaborative work within VOs leveraging the emerging convergence of Web Services and Grid technologies will be constructed. A realisation of the TrustCoM framework will be delivered by means of open-standards Web Services based specifications and a reference implementation. Validation will take place within industrial strength test-beds in the areas of collaborative engineering and provision of ad hoc, aggregate electronic services. Despite the differences of technologies used in the two projects, the set of issues is largely the same in both cases - location of the partners and services, selection of the appropriate services, secure collaboration, business rules enactment, etc. This similarity helps to transfer the knowledge and apply it in a new context.

The architecture described in this paper does not explicitly address RBVO management by assuming the overall information about VO to be a sum of the knowledge shared by involved participants and the operation of such community is governed only by business collaborations. While this approach simplifies the architecture and its instantiation considerably, there might be cases when a virtual community needs to be managed more explicitly and the participants need to commit to the policies of such virtual formation as a whole before they can enter bilateral or multi-lateral collaborations. The drivers for such approach may include: higher security and trust requirements, more sensitive business area, stronger audit requirements, etc.

\section{ACKNOWLEDGEMENTS}

The results presented here are partially funded by the European Commission under contract IST-2001-33251 through the project LAURA. The future research ideas arise from the work in TrustCoM project, funded by the European Commission under contract IST-2003-01945. The authors would like to thank members of respective partner organisations of both projects for their valuable input and help. 


\section{REFERENCES}

1. Aldrich, D.F. (1999), Mastering The Digital Marketplace, John Wiley, Chichester

2. Benjamin, R.I., Malone,W.T. \& Yates, J. (1986), Electronic Markets And Electronic Hierarchies. CISR Working Paper No 137. Centre For Information Systems Research, Sloane School of Management, Massachusetts Institute Of Technology.

3. Bovet, D. \& Martha, J., (2000), Value Nets: Breaking the Supply Chain To Unlock Hidden Profits, John Wiley, Chichester

4. Bressler, S. E. \& Grantham, C.E., (2000) Communities of Commerce, McGraw Hill, new York

5. Cash,J.I. Jr, (1985), Interorganisational Systems: An Information Society Opportunity Or Threat? The Information Society, 3(3), 199-228.

6. Cash, J.I. \& Konsynski, B.R. (1985), IS Redraws Competitive Boundaries, Harvard Business Review, March-April, 134-142

7. Choudhury, V. (1997), Strategic Choices in the Development of Inter-Organizational Information Systems, Information Systems Research, Vol 8, No 1

8. Davidow, W.H \& Malone, S.M. (1992), The Virtual Corporation - Structuring And Revitalizing The Corporation For The 21st Century, Harper Collins, New York

9. Dimitrakos, T. et al (2004), TrustCoM - A Trust and Contract Management Framework enabling Secure Collaborations in Dynamic Virtual Organisations. ERCIM News No. 59, pp 59-60, Sophia Antipolis, France. http://www.ercim.org/publication/Ercim_News/enw59/dimitrakos2.html (accessed December 2004).

10. Dubray, J.J. (2001). OAGIS Implementation Using the ebXML CPP, CPA and BPSS specifications v1.0. hitp/www.openapplications.org/downlods/whilepapers/frameworlis (accessed December 2004)

11. EbXML. (2003). The ebXML Framework, http//www ebxmlorg (accessed November 2004)

12. Flebowitz, M. (2002). OAGIS 8.0: Practical Integration meets XML Schema. XML Journal, Volume 03, Issue 09. http//www openapplications.org/news/articles/XMIJ-Septo2OACIS8PracticalntegrationMcets XMLScherna pd (accessed November 2003)

13. Hagel, J. \& Armstrong, A. (1997), Net.Gain: Expanding Markets Through Virtual Communities, Harvard Business School Press, Harvard

14. JXTA (2004). JXTA Technology: Creating Connected Communities. htw//wwwjxta org/proiectwww/docs/IXTA-Exec-Briefpdf (accessed February 2005)

15. LAURA (2003). EU IST-2001-33251 project. htip//www.lauraproject.org

16. Lee, K.J. , Peer-to-Peer Electronic Commerce and Intelligent Systems, htip//space postechac.kr/vod/s01/204/ppt204/ppt1204.pdf. (Accessed August 2004)

17. Lethbridge, N., (2001), An I-Based Taxonomy of Virtual Organisations and the Implications for Effective Management, Developing Effective Organisations, Vol 4, No 1, pp.16-24

18. Marjanovic, O. (2002), Supporting Co-ordination in Dynamic Virtual Enterprises, Proceedings of $15^{\text {th }}$ Bled Electronic Commerce Conference, e-Reality: Constructing the e-Economy, Bled, Slovenia, June 17-19, pp. 712-726,

19. Nikoleris, G. \& Johansson, N. (2003), Managing the Exchange of Data in the Extended Enterprise, Proceedings of $10^{\text {th }}$ ISPE International Conference, Madeira, 28-30 July, pp. 317-321

20. OFBiz. (2003). The Open For Business Project, hitp:/www obix org (accessed November 2003)

21. Rayport J.F. \& Sviolka J.H. (1994) Managing in the Marketspace, Harvard Business Review, 72(6) (Nov-Dec) pp. 140-150

22. Rhodes, E. \& Carter, R. (1994), Engineering The Virtual Enterprise, Paper presented at Research Forum, 5th World Congress Of EDI Users, Brighton, June 14-15

23. Rowell, M. (2002). OAGIS: A Canonical Business Language. XML Journal, Volume 03, Issue 09.

24. Saabeel, W. et al. (2002), A Model Of Virtual Orgasnization: A Structure And Process Perspective, Electronic Journal Of Organizational Virtualness, Vol. 4, No. 1

25. Sieber, P., Griese, J., (1999), Virtual Organizations As Power Asymmetrical Networks, Organizational Virtualness And E-Commerce, 2 International VoNet Workshop, Zurich

26. Silva, J.P., Putnik, G.D. \& Cunha,M.M. (2003), Technologies for Virtual Enterprise Integration, published in Business Excellence: Performance measures, Benchmarking and Best Practices in New Economy, Univ of Minho Press, pp.706-712

27. Timmers, P., (1999), Electronic Commerce: Strategies and Models for B2B Trading, John Wiley, Chichester 